\title{
Solving Ordinary Differential Equations (ODEs) Using Least Square Method Based on Wang Ball Curves
}

\author{
Abdul Hadi Bhatti ${ }^{*}$, Sharmila Binti Karim \\ Department of Mathematics \& Statistics, School of Quantitative Sciences, Universiti Utara Malaysia, \\ 06010 UUM Sintok, Kedah, Malaysia
}

Received September 29, 2021; Revised November 25, 2021; Accepted December 13, 2021

\begin{abstract}
Cite This Paper in the following Citation Styles
(a): [1] Abdul Hadi Bhatti, Sharmila Binti Karim, "Solving Ordinary Differential Equations (ODEs) Using Least Square Method Based on Wang Ball Curves," Mathematics and Statistics, Vol. 10, No. 1, pp. 74 - 87, 2022. DOI: 10.13189/ms.2022.100106.
\end{abstract}

(b): Abdul Hadi Bhatti, Sharmila Binti Karim (2022). Solving Ordinary Differential Equations (ODEs) Using Least Square Method Based on Wang Ball Curves. Mathematics and Statistics, 10(1), 74 - 87. DOI: 10.13189/ms.2022.100106.

Copyright $\bigcirc 2022$ by authors, all rights reserved. Authors agree that this article remains permanently open access under the terms of the Creative Commons Attribution License 4.0 International License

\begin{abstract}
Numerical methods are regularly established for the better approximate solutions of the ordinary differential equations (ODEs). The best approximate solution of ODEs can be obtained by error reduction between the approximate solution and exact solution. To improve the error accuracy, the representations of Wang Ball curves are proposed through the investigation of their control points by using the Least Square Method (LSM). The control points of Wang Ball curves are calculated by minimizing the residual function using LSM. The residual function is minimized by reducing the residual error where it is measured by the sum of the square of the residual function of the Wang Ball curve's control points. The approximate solution of ODEs is obtained by exploring and determining the control points of Wang Ball curves. Two numerical examples of initial value problem (IVP) and boundary value problem (BVP) are illustrated to demonstrate the proposed method in terms of error. The results of the numerical examples by using the proposed method show that the error accuracy is improved compared to the existing study of Bézier curves. Successfully, the convergence analysis is conducted with a two-point boundary value problem for the proposed method.
\end{abstract}

Keywords Wang Ball Curves, Ordinary Differential Equations, Least Square Methods, Residual Function

\section{Introduction}

In real-world applications like various fields of engineering and computer science, many problems involve mathematical models which contain an ordinary differential equation (ODE). Most ODEs' problems, which evolve in such applications, are difficult to achieve an exact or analytical solution due to the complexity of exponent, trigonometric and polynomial functions. Many research scholars have come up with the approximate solution of ODEs in the form of polynomial or piecewise polynomial functions by employing various numerical methods such as series, finite element method, shooting, segmentation and $\operatorname{LSM}[5,8,30,32,41-42,48]$.

Polynomial and piecewise polynomial functions are obtained as an approximate solution by using the control points of Bézier curves. The Bézier curve's control points have computational advantages in terms of error accuracy $[1,8,30,48]$. Moreover, a useful strategy is to increase the Bézier curve's degree if the approximate solution is not on the desired level and contrarily, it can be decreased to reduce the extra calculation burden if the solution is already on the desired level [2]. Since the Bézier curve's control points' structure becomes an important geometric feature of the Bézier shape, computational performance is improved while solving ODEs based on the control points $[2,30]$. Thus, the Bézier parametric curve is an outstanding technique of getting the solution to a problem that involves parametric surfaces and curves.

Since the 1970s, some scholars have been establishing 
the basis functions of generalized Ball curves in their studies. According to the Ball curve's history, in 1974, Alan Ball developed and introduced the cubic Ball curve's basis functions for conic lofting surface program CONSURF while working at the British Aircraft Corporation (BAC) $[4,7,11]$. In 1987, Wang increased the degrees of the cubic Ball basis functions to the generalized higher level [13]. Further in 1989, Said proposed the general odd degree to the cubic Ball basis functions to generalize the Ball curves [12]. Then, in 1991, Goodman and Said's studies showed that the basis functions of generalized Ball curves with the odd degree are totally positive and the preservative properties of the curves are uniform between the generalized Ball curves and Bézier curves $[9,12,21]$. Furthermore, in another study, they proved that the degree reducing and raising of the generalized Ball curves is more suitable compared to that of the Bézier curves [15]. Since then, the degree raising and lowering of the Ball curve's control points was found to be a wonderful geometric instrument for obtaining the exact shape of the curve - the computations are regarded as an attractive technique $[2,12,16-17,19]$. Therefore, by using the increment and decrement of degrees, generalized Ball curves were found to be more efficient than Bézier curves while applying the de Casteljau algorithm as well as a recursive algorithm $[12,14]$.

The study of the Ball curve inspired many scholars to explore its accurate shape by increasing or decreasing its degree through theoretical computation [3, 12, 15-20]. Hence, the generalized Ball curves extended to Wang Ball curves, Said Ball curves, DP Ball curves and rational Ball curves with higher degree $n$ polynomials $[10,12-13,16]$. The highest degree of Wang Ball curves, Said Ball curves, DP Ball curves and rational Ball curves can be found by overlying their control points [22].

The mathematical of the Ball curve's basis function of a cubic polynomial curve is as follows:

$$
(1-x)^{2}, 2 x(1-x)^{2}, 2 x^{2}(1-x), x^{2}
$$

Two advantages of the generalized Ball curves are observed: the Ball function's degree three polynomial, combined with their internal control points, can be reduced to the Bézier function's degree two polynomials; and the computational competence is found to be better than that of the Bézier curves' while using the generalized Ball curves [12].

At any rate, the Wang Ball curve is proposed to approximate the solution of ODEs. Since the Ball curves sparked global interest, research scholars started to find the approximate solutions of higher order ODEs by developing the algorithms through different numerical methods such as the reduction method [24-27]. However, there are many hurdles found in the reduction method such as lengthy writeup because of its numerous iterations, taking more time while developing the program on a computer and the error accuracy affected because of its computational load
[24-25]. Hence, research scholars created the direct method to overcome these hurdles while solving higher order ODEs approximately [25]. The direct method has the capability to efficiently solve the higher order ODEs without reducing them to the first order ODEs [41, 43-46].

The Least Squares Methods (LSM) is one of the direct methods. The better accuracy in terms of error for the approximate solution of higher order ODEs can be obtained by using LSM through the polynomial or piecewise polynomial functions [32]. Meanwhile, many researchers used LSM in solving different types of differential equations approximately [33-40]. The LSM is employed based on the control points of Bézier curves by developing the least square objective function for the discretization of integrals to improve the approximate solutions of higher order ODEs [29]. Furthermore, the LSM was applied for solving two-point boundary value problems through two different schemes: degree raising and subdivision. The convergence analysis of the control point-based methods LSM to the two-point boundary value problem showed that the LSM is a reliable method [30].

Lyche and Morken [31] stated that the LSM is an efficient and simple method when employed to solve higher order ODEs approximately. From past reviews, the application of the LSM to find the approximate solution of higher order ODEs based on the Bézier curve's control points, the result is only satisfied, but not on the required level in terms of error [30, 36, 39-40, 47].

The structural preservative properties between the Bézier curves and generalized Wang Ball curves are similar; furthermore, the Wang Ball curves based on the control points have yet to be investigated to find the approximate solution of higher order ODEs.

Thus, we propose the LSM to solve higher order ODEs by exploring the control points of Wang Ball curves to improve the accuracy in terms of error. The remainder of this paper is structured as follows: the Bézier curve's representation in Section 2, the representations of Wang Ball curves along with their properties are briefly discussed in Section 3 while in Section 4, the new method is proposed for solving ODEs approximately by investigating the control points of Wang Ball curves; thereafter in Section 5, numerical examples are provided to demonstrate the newly proposed method; furthermore, in Section 6, the convergence of the proposed method for the two-point value problem is analyzed; Finally, in Section 7 the conclusion is presented.

\section{Bézier Curves Representations}

The Bézier curves of degree $m$ and $(m+1)$ control points $\left\{\boldsymbol{b}_{\boldsymbol{j}}\right\}_{\boldsymbol{j}=\mathbf{0}}^{\boldsymbol{m}}$ can be defined as follows [30]:

$$
B(x)=\sum_{j=0}^{m} b_{j} B_{j}^{m}\left(\frac{x-a}{b-a}\right), \mathrm{a} \leq x \leq \mathrm{b}
$$

where $b_{j}$, the Bézier coefficient called the Bézier control 
point and the Bézier curves Polynomials $B_{j}^{m}(x)$ over the interval $\mathrm{a} \leq x \leq \mathrm{b}$ is as follows:

$$
B_{j}^{m}\left(\frac{x-a}{b-a}\right)=\left(\begin{array}{c}
m \\
j
\end{array}\right)\left(\frac{x-a}{b-a}\right)^{j}\left(\frac{b-x}{b-a}\right)^{m-j}
$$

In particular

$$
B(x)=\sum_{j=0}^{m} b_{j} B_{j}^{m}(x), 0 \leq x \leq 1
$$

Where

$$
B_{j}^{m}(x)=\left(\begin{array}{c}
m \\
j
\end{array}\right) x^{j}(1-x)^{m-j}
$$

If $B(x)$ be a vector valued polynomial, then $B(x)$ is a parametric Bézier curve. The Bézier curve's control polygon involves the line segments $b_{j}-b_{j+1}$, where $j=0,1,2, \ldots, m-1$. If $B(x)$ be scalar valued function, then the function $y=B(x)$ called the explicit Bézier curve presented by $(x, B(x))$.

\section{Wang Ball Curves Representations}

The Wang Ball curves with degree $m$ and $(m+1)$ control points $\left\{\boldsymbol{w}_{\boldsymbol{j}}\right\}_{\boldsymbol{j}=\mathbf{0}}^{\boldsymbol{m}}$ are as follows [13]:

$$
A(x)=\sum_{j=0}^{m} w_{j} A_{j}^{m}(x), 0 \leq x \leq 1
$$

where the Wang Ball Polynomials $A_{j}^{m}(x)$ are defined as follows:

$$
A_{j}^{m}(x)=\left\{\begin{array}{l}
(1-x)^{2+j}(2 x)^{j}, \text { for } 0 \leq j \leq \frac{m-3}{2} \\
(1-x)^{\frac{m+1}{2}}(2 x)^{\frac{m-1}{2}}, \text { for } j=\frac{m-1}{2} \\
(2(1-x))^{\frac{m-1}{2}}(x)^{\frac{m+1}{2}}, \text { for } j=\frac{m+1}{2} \\
(2 x(1-x))^{m-j}(x)^{m-j+2}, \text { for } \frac{m+3}{2} \leq j \leq m
\end{array}\right.
$$

when $m$ is odd and

$$
\left\{\begin{array}{l}
(1-x)^{2+j}(2 x)^{j}, \text { for } 0 \leq j \leq \frac{m}{2}-1 \\
(2 x(1-x))^{\frac{m}{2}}, \text { for } j=\frac{m}{2} \\
(2(1-x))^{m-j}(x)^{m-j+2}, \text { for } \frac{m+2}{2} \leq j \leq m
\end{array}\right.
$$

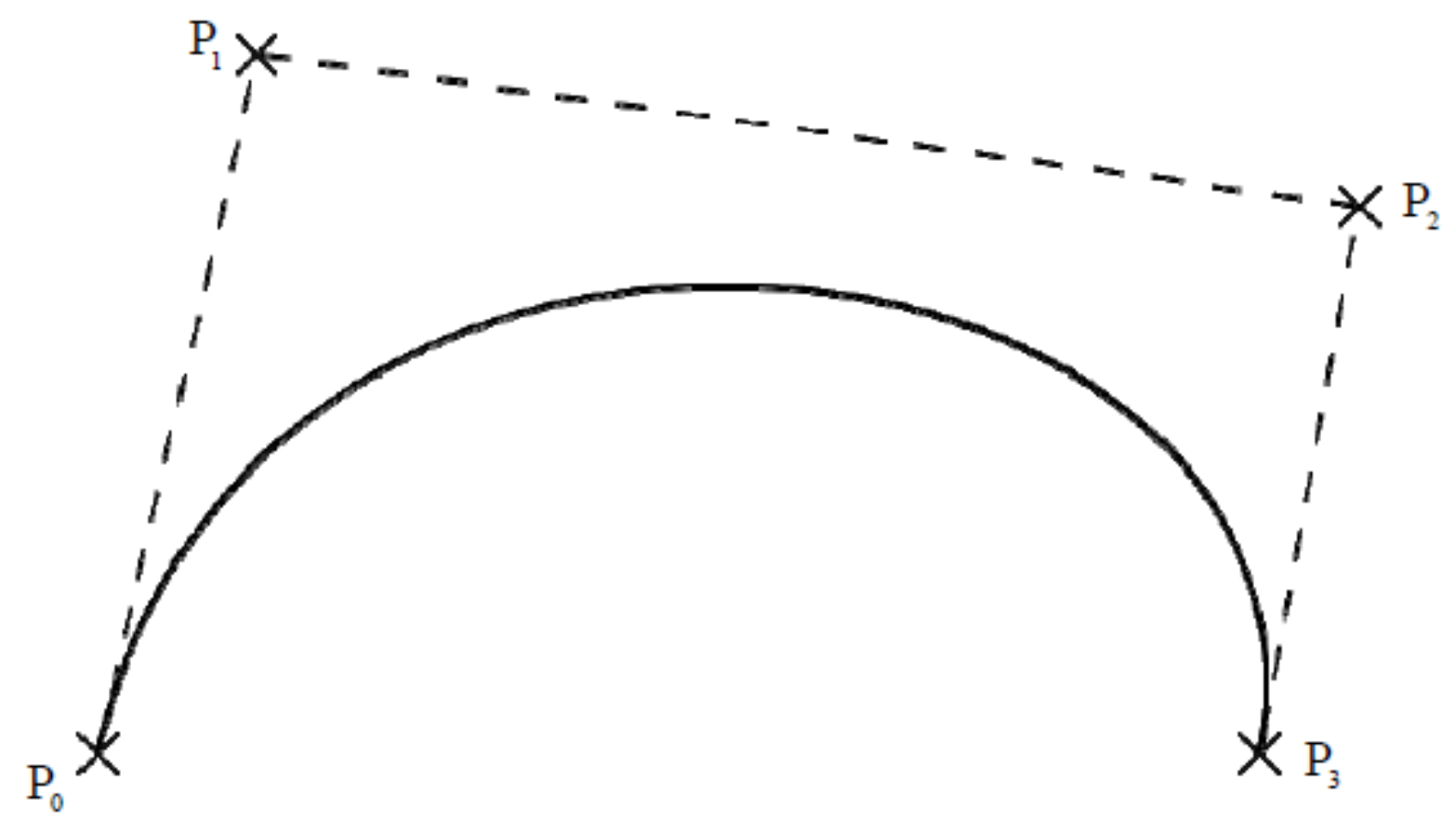

Figure 1. The Bézier curve of degree 3 and its control polygon. 
The graphical representations of the Wang Ball curve's basis function with degrees 2, 3 and 4 are shown below in figures 2,3 , and 4 , respectively.

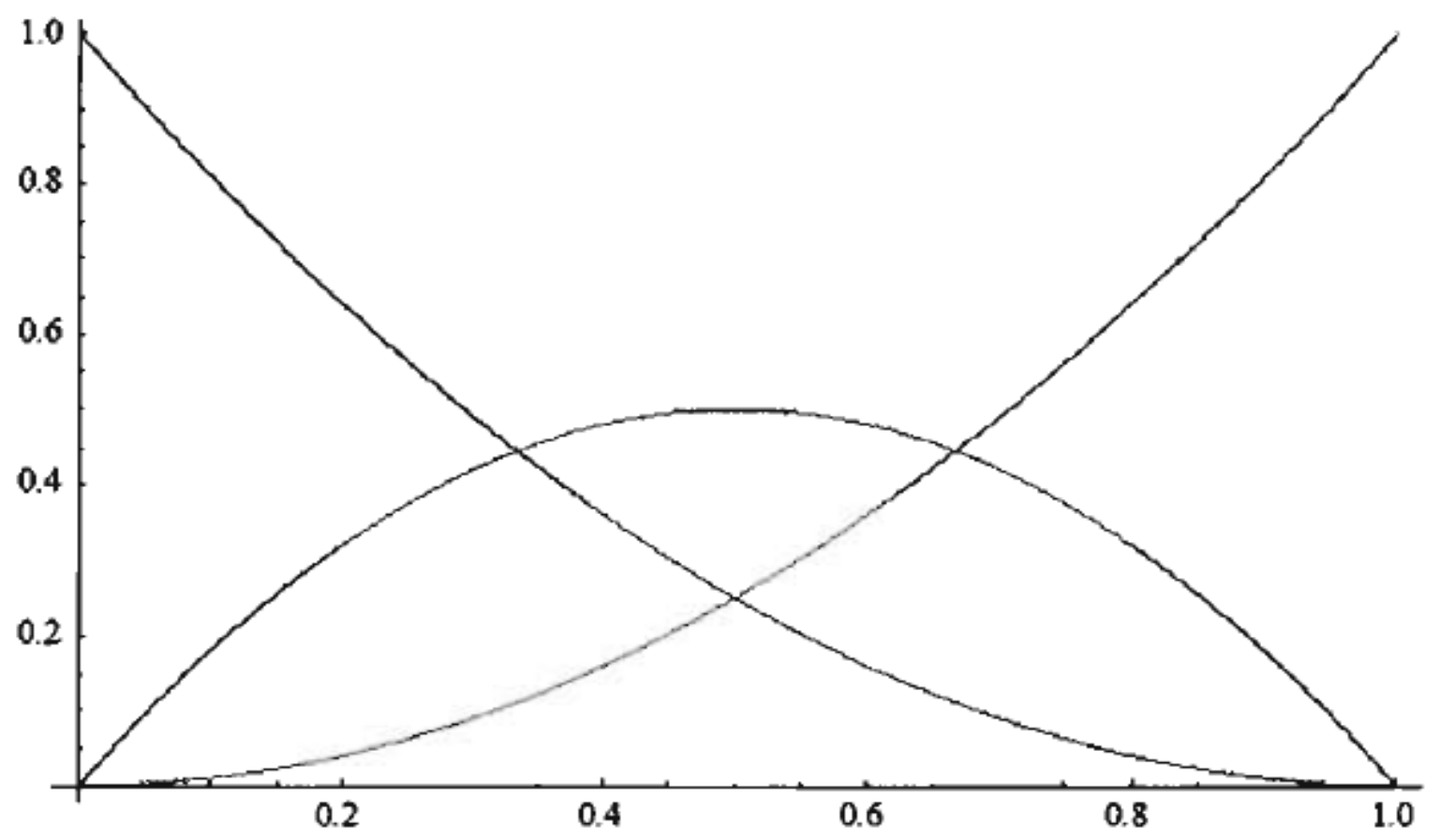

Figure 2. Basis function of Wang Ball with degree 2

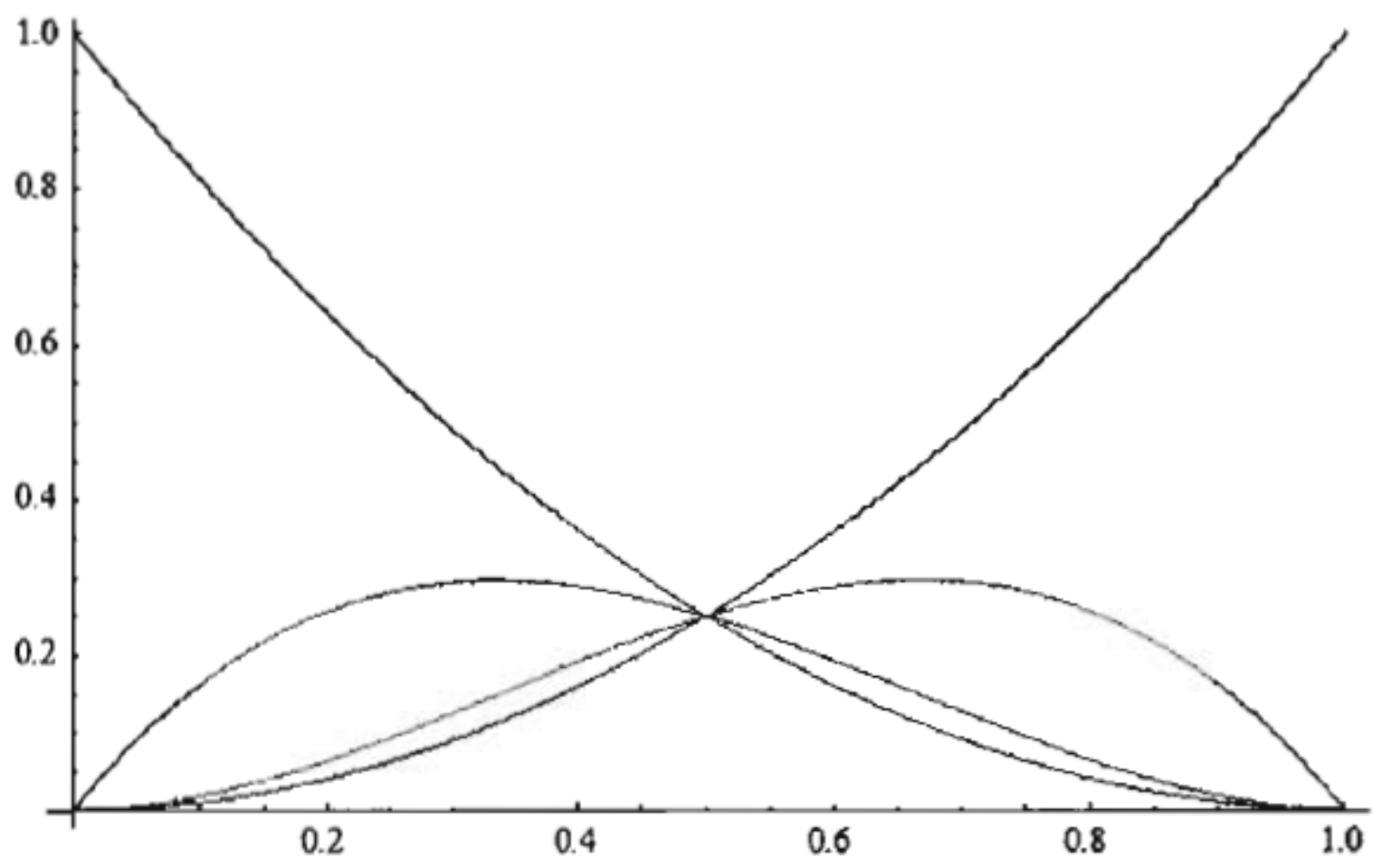

Figure 3. Basis function of Wang Ball with degree 3 


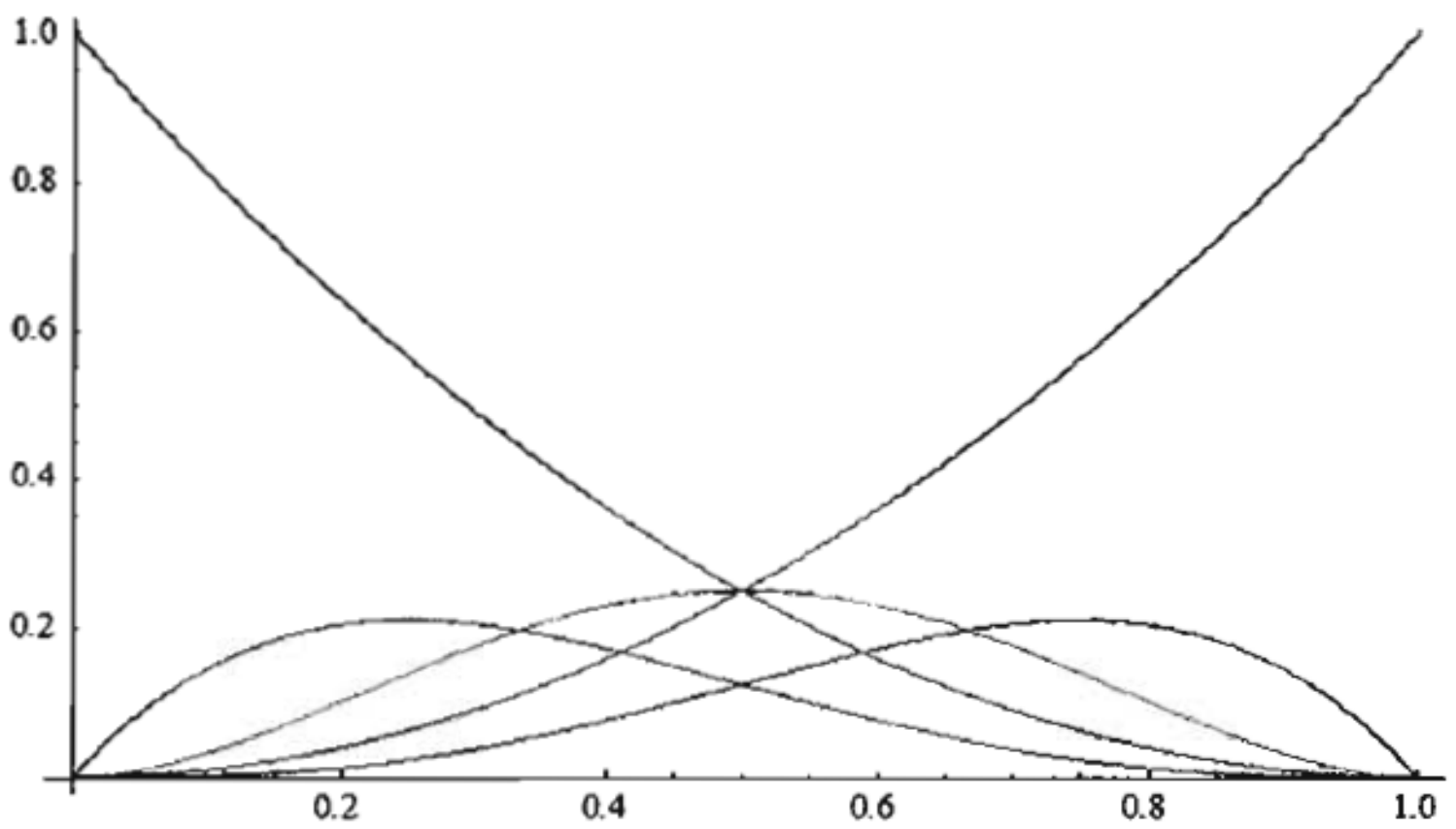

Figure 4. Basis function of Wang Ball with degree 4 are:

The properties of the Wang Ball curve's basis functions

i. The basis function of Wang Ball curves is non-negative.

$$
A_{j}^{m}(x) \geq 0, \text { for all } j=0,1,2, \ldots, m
$$

ii. The partition of the Wang Ball curve's basis function is unity.

$$
\sum_{j=0}^{m} A_{j}^{m}(x)=1
$$

The above two properties, non-negativity, and unity of partition of Wang Ball curve's basis function in the above equations (3.4) and (3.5) satisfied the convex combination of the control points, as well as the curve of the Wang Ball basis function, lie in the convex hull with its control polygon [16-17].

\section{Least Squares Method for the Approximate Solution of ODEs}

\subsection{The Wang Ball curve's Control Points-Based Approach}

Since then, the Wang Ball curve has more computational competence than the Bézier curve and the constructional properties of the shape preservative are similar between the Wang Ball curve basis function and Bézier curve basis function. The control point based approach is proposed to represent the approximate solution of ODEs in the form of the Wang Ball curve by using the LSM. We take the sum of squares of the control points of Wang Ball curves of the residual function $R(x)=L g(x)-f(x)$ to compute the residual error by constructing the objective function with the LSM. By minimizing the objective function, if the residual error is equal to zero then the residual function is also equal to zero, which implies that the approximate solution is equal to the exact solution of ODEs.

To demonstrate the proposed method, the following IVP and BVP are considered for the approximate solution:

$L g(x)=\sum_{j=0}^{2 m} \varphi_{j}(x) g^{(j)}(x)=f(x), \quad 0 \leq x \leq 1$

with initial value problem as follows:

$$
g(0)=g_{0}, g^{(j)}(0)=g_{j}, j=1,2,3, \ldots, m-1
$$

and with boundary value problem as follows:

$$
\begin{gathered}
g^{(j)}(0)=g_{j}, g^{(j)}(1)=z_{j} \text { with } g^{(0)}=g, \\
j=0,1,2,3, \ldots, m-1
\end{gathered}
$$

where:

$$
L=\sum_{j=0}^{2 m} \varphi_{j}(x) \frac{d^{j}}{d x^{j}}
$$

is a differential operator of $(2 m)^{\text {th }}$ order along with the $\varphi_{j}(x)$, polynomial coefficients in terms of $x$, and $f(x)$, the polynomial function in terms of $x$.

The aim of this paper is to develop a generalized method to solve the higher order ODEs approximately by computing the residual error through minimization of the objective function based on the control points of Wang Ball curves in the residual function $R(u)=L g(x)-f(x)$. Thereafter, an algorithm for the approximate solution of 
ODEs in the form of polynomial function $g(x)$, satisfying the conditions of IVPs (4.2) and BVPs (4.3), is developed.

The algorithm to solve the ODEs by using the LSM through computing the control points of Wang Ball curves is shown in the following steps:

Step 1: Suppose that a degree $m$ and symbolically express $A(x)$, the approximate solution of ODEs in the form of Wang Ball curves with degree $m$, respectively, is:

$$
A(x)=\sum_{j=0}^{m} w_{j} A_{j}^{m}(x), 0 \leq x \leq 1
$$

Where $\quad(m+1)$ control points $\left\{w_{j}\right\}_{j=0}^{m}=w_{0}, w_{1}, w_{2}, \ldots, w_{m}$ are to be determined.

Step 2: Determining the residual function $R_{w}(x)=$ $L g(x)-f(x)$ of the Wang Ball curves by substituting the solution $A(x)$ into the IVPs and BVPs of ODEs in the form of Wang Ball curves. The polynomial

$$
R_{w}(x)=\sum_{j=0}^{k} c_{w j} A_{j}^{m}(x)
$$

can be expressed with degree $\leq k$ in the form of Wang Ball curves.

We note that $k=\max _{j}\left\{m-j+\operatorname{deg}\left(\varphi_{j}\right), \operatorname{deg}(f(x))\right\}$ and where the linear functions $c_{w j}$ are control points in terms of unknowns $w_{j}$ and $j=0,1,2,3, \ldots \ldots \ldots, k$. These functions will be found by implementing the techniques of multiplication, degree elevation and reduction, and differentiation of the Wang Ball curves.

Step 3: Constructing the objective functions for Wang Ball curves.

$$
F_{w}=\sum_{j=0}^{k} c_{w j}^{2} w_{j}
$$

Then also let $F_{w}$ be a function of $w_{j}=w_{0}, w_{1}, w_{2}, \ldots, w_{m}$.

Step 4: Solving the constrained optimization problems for $w_{j}$

$$
\begin{gathered}
\min F_{w}=\sum_{j=0}^{k} c_{w j}^{2} w_{j} \\
\text { s.t } g^{j}(0)=g_{j}, j=0,1,2,3, \ldots, m-1
\end{gathered}
$$

Or

$$
g^{j}(0)=g_{j} \text { and } g^{j}(1)=z_{j}, j=0,1,2,3, \ldots, m-1
$$

The Lagrange Multiplier method or any other suitable method will be employed which can reduce this constrained optimization problem into a problem of solving a system of linear equations.

Step 5: By replacing the minimum solutions which we find in last Step 4 back into the solutions $A(x)$ into equation (4.4) in Step 1, which is the form of Wang Ball curves, the approximate solutions of the ODEs will be found.

\subsection{The Degree Raising Strategy}

Using the control point-based approach in Section 3.1 allows us to obtain the approximate solution of higher order ODEs using LSM. By replacing all the control points $w_{j}$ into the objective function $F_{w}$, we can either obtain the Euclidean norm of the Wang Ball curve's control points, or according to the convex hull property of Wang Ball curves, obtain the upper bound on the residual function $R_{w}(x)$ from the values of $c_{j}$ as follows:

$$
\left|R_{w}(x)\right| \leq \max _{j}\left\{\left|c_{j}\right|\right\}, \quad 0 \leq x \leq 1
$$

The approximate solution is not on the desired level if the above values in (4.5) sufficiently not small. Then, by raising the degree of approximate solution and again running the Wang Ball curve's control point base approach using the LSM in Section 4.1, an approximate solution will be improved in terms of error accuracy.

\section{Numerical Examples}

The proposed method is demonstrated in solving two numerical examples by computing the control points of Wang Ball curves using LSM. The resulting accuracy can be verified in terms of error and efficiency in terms of computation.

\subsection{Problem 1: Boundary Value Problem of Second Order ODE [30].}

Let us consider a simple numerical example, BVP of second order ODE, to demonstrate and explain the newly proposed method.

$y^{\prime \prime}(x)-6 x=0$, with $y(0)=0, y(1)=1$.

$y_{e}(x)=x^{3}$ is the exact solution for this IVP of second order ODE.

First Step 2: Determining A polynomial of degree 2 will be obtained to solve the IVP of order 2 ODE through LSM based on the Wang Ball curve's control points. Suppose that the following Wang Ball curve $y_{a}=A(x)$ of degree 2 is the approximate solution of this numerical example.

$$
\begin{gathered}
y_{a}=A(x)=\sum_{j=0}^{2} A_{j}^{m}(x) w_{j} \\
=w_{0} A_{0}^{2}(x)+w_{1} A_{1}^{2}(x)+w_{2} A_{2}^{2}(x) \\
y_{a}(x)=A_{a}(x)=w_{0}(1-x)^{2}+w_{1} 2 x(1-x)+ \\
+w_{2} x^{2}
\end{gathered}
$$

Second step: An approximate solution $y_{a}=A(x)$ (Equation 4.1) will be replaced into the given second order ODE and obtained the residual function as follows: 


$$
\begin{gathered}
R_{w}(x)=2 w_{0}-4 w_{1}+2 w_{2}-6 x \\
R_{w}(x)=\left(2 w_{0}-4 w_{1}+2 w_{2}\right) A_{0}^{2}(x)+ \\
+\left(2 w_{0}-4 w_{1}+2 w_{2}-6 x\right) A_{1}^{2}(x)
\end{gathered}
$$

Third step: Constructing an objective function for the Wang Ball curves as follows:

$$
\begin{gathered}
F_{w}\left(w_{0}, w_{1}, w_{2}\right)=\left(2 w_{0}-4 w_{1}+2 w_{2}\right)^{2}+ \\
+\left(2 w_{0}-4 w_{1}+2 w_{2}-6\right)^{2}
\end{gathered}
$$

Fourth step: Computing the control points of Wang Ball curves as $w_{0}=0, w_{1}=0$ and $w_{2}=1$ with the initial conditions $y(0)=0, y(1)=1$ and by solving the optimization problem through minimizing the objective function.

Fifth step: Substituting the control points of Wang Ball curves $w_{0}=0, w_{1}=0$ and $w_{2}=1$ back into our assumed approximate solution in Equation 5.1. Hence, the approximate solution of the given 2nd order ODE arrives as $y_{a}(x)=A_{a}(x)=x^{2}$. The highest error 0.002375 is noticed between the exact and approximate solutions at $x=0.05$ in the interval $[0,1]$ which is acceptable.

\subsection{Example 2: A Boundary Value Problem of a} Fourth-Order ODE Involved in a Mathematical Model for Transversal Bending of Clamped Beam [30]

$$
\left(\left(x^{2}+1\right) y^{\prime \prime}(x)\right)^{\prime \prime}=x+1,0<x<1,
$$

with $y(0)=y(1)=0$, and $y^{\prime}(0)=y^{\prime}(1)=0$.

The exact solution of above BVP of $4^{\text {th }}$ degree ODE is given by:

$$
\begin{aligned}
& y_{e}(x)=\left[336 x \arctan (x)-36 \pi x^{2}+9 \pi^{2} x^{2}\right. \\
&+36(\ln (2))^{2} x^{2}-4 \pi x^{3}+\pi^{2} x^{3} \\
&+4(\ln (2))^{2} x^{3}-44 \pi x \\
&+168(\ln (2)) x \\
&-168(\ln (2)) \arctan (x) \\
&+44 \pi \arctan (x)-168\left(\ln \left(1+x^{2}\right)\right) \\
&+44(\ln (2))\left(\ln \left(1+x^{2}\right)\right) \\
&+42 \pi\left(\ln \left(1+x^{2}\right)\right)-84 \pi x \arctan (x) \\
&-88(\ln (2)) x \arctan (x) \\
&+22 \pi x\left(\ln \left(1+x^{2}\right)\right) \\
&\left.-84(\ln (2)) x\left(\ln \left(1+x^{2}\right)\right)\right] \\
& / 36\left(4(\ln (2))^{2}+\pi^{2}-4 \pi\right) .
\end{aligned}
$$

First the approximate solution demonstrated by existing approach LSM based on the Bézier curve's control points with degree 5 and degree 8 .
Suppose that the approximate solution of 4 th order ODE is the Bézier curve of degree 5 as follows:

$$
\begin{gathered}
y_{a}(x)=\mathrm{B}(x)=\sum_{j=0}^{5} b_{j} B_{j}^{m}(x) \\
y_{a}(x)=B(x)=b_{0} B_{0}^{5}(x)+b_{1} B_{1}^{5}(x)+b_{2} B_{2}^{5}(x)+ \\
+b_{3} B_{3}^{5}(x)+b_{4} B_{4}^{5}(x)+b_{5} B_{5}^{5}(x) \\
y_{a}(x)=B(x)=b_{0}(1-x)^{5}+5 b_{1} x(1-x)^{4}+ \\
+10 b_{2} x^{2}(1-x)^{3}+10 b_{3} x^{3}(1-x)^{2}+ \\
+5 b_{4} x^{4}(1-x)+b_{5} x^{5}
\end{gathered}
$$

The Bézier curve's control points calculated through minimizing the objective function applying LSM along the given boundary conditions $y(0)=y(1)=0, y^{\prime}(0)=$ $y^{\prime}(1)=0$ then by solving the constrained optimization problem. The Bézier curve's control points values are $b_{0}=0, b_{1}=0, b_{2}=0.0043, b_{3}=0.0033, b_{4}=0$, and $b_{5}=0$. The approximate solution based on the Bézier curve of degree 5 is

$$
\begin{aligned}
y_{a}(x)= & B(x)=0.043 x^{2}(1-x)^{3}+ \\
& +0.033 x^{3}(1-x)^{2}
\end{aligned}
$$

And now suppose that the Bézier curve of degree 8 is the approximate solution of 4 th order ODE is the as follows:

$$
\begin{gathered}
y_{a}(x)=\mathrm{B}(x)=\sum_{j=0}^{8} b_{j} B_{j}^{m}(x) \\
y_{a}(x)=B(x)=b_{0} B_{0}^{8}(x)+b_{1} B_{1}^{8}(x)+b_{2} B_{2}^{8}(x)+ \\
+b_{3} B_{3}^{8}(x)+b_{4} B_{4}^{8}(x)+b_{5} B_{5}^{8}(x)+b_{6} B_{6}^{8}(x)+ \\
+b_{7} B_{7}^{8}(x)+b_{8} B_{8}^{8}(x) \\
y_{a}(x)=B(x)=b_{0}(1-x)^{8}+8 b_{1} x(1-x)^{7}+ \\
28 b_{2} x^{2}(1-x)^{6}+56 b_{3} x^{3}(1-x)^{5}+70 b_{4} x^{4}(1- \\
x)^{4}+56 b_{5} x^{5}(1-x)^{3}+28 b_{6} x^{6}(1-x)^{2}+ \\
8 b_{7} x^{7}(1-x)+b_{8} x^{8}
\end{gathered}
$$

By minimizing the objective function using LSM along with the given boundary conditions $y(0)=y(1)=0$, $y^{\prime}(0)=y^{\prime}(1)=0$, found the control points of Bézier curve. The values of the control points for Bézier curve are $b_{0}=0 \quad, \quad b_{1}=0 \quad, \quad b_{2}=0.0018082143$, $b_{3}=0.0036007143, \quad b_{4}=0.0040078571, \quad b_{5}=$ $0.0030312500, b_{6}=0.0013717857, b_{7}=0$, and $b_{8}=0$. Thus, the approximate solution based on the Bézier curve of degree 8 is as follows:

$$
\begin{gathered}
y_{a}(x)=B(x)=0.05063 x^{2}(1-x)^{6}+ \\
+0.20164 x^{3}(1-x)^{5}+0.280549997 x^{4}(1-x)^{4}+ \\
+0.16975 x^{5}(1-x)^{3}+0.038409999 x^{6}(1-x)^{2}(5.5)
\end{gathered}
$$

The graph of the approximate of degree 5 , degree 8 and exact solution is presented in the following figure 5 . 


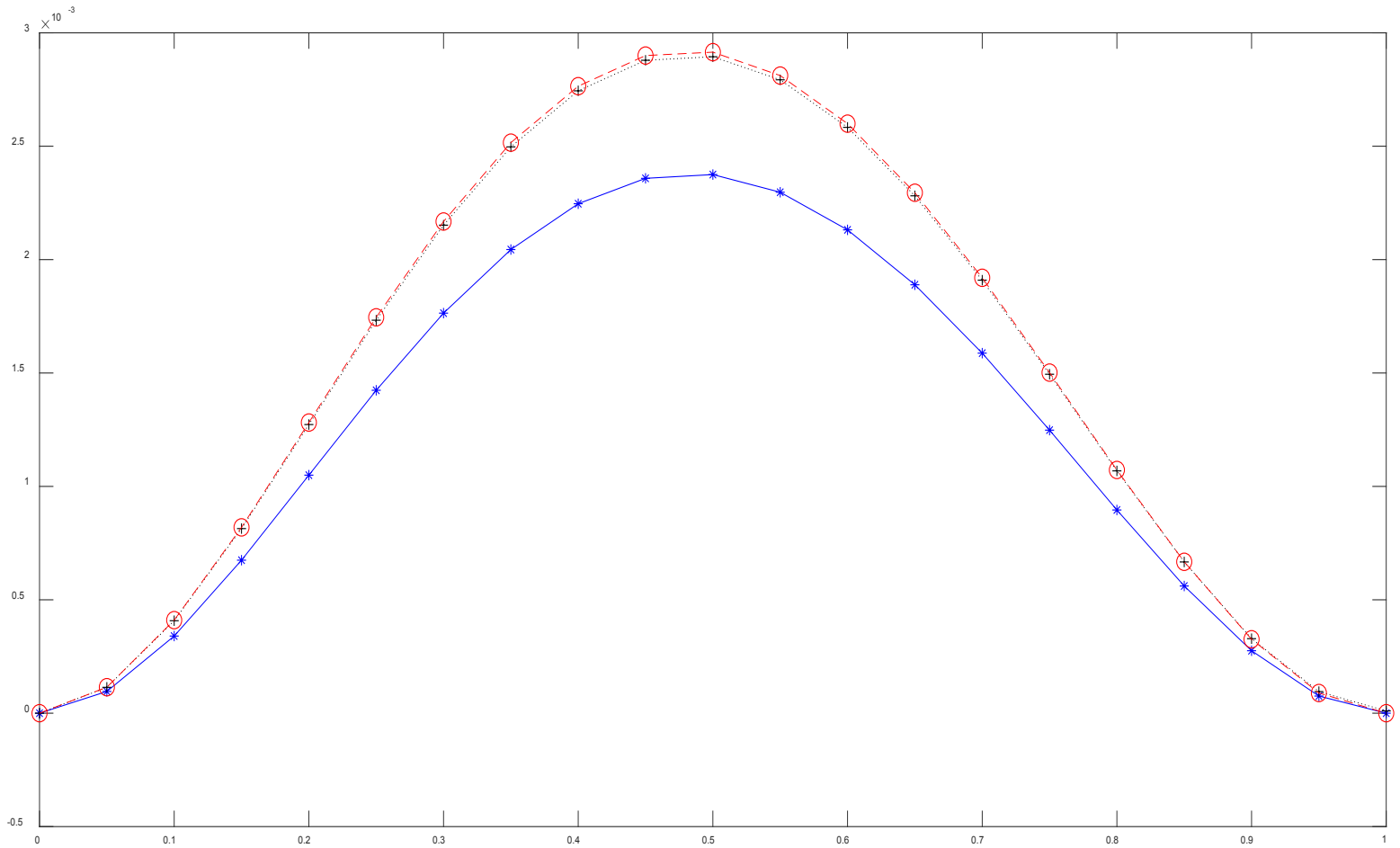

Figure 5. xact $y_{e}(x)$ (dashed with circles in red colour), the approximate solution $y_{a}(x)$ with Bézier curve curve's basis function of degree 5 (dashed with stars in blue colour) and degree 8 (dotted with plus in black colour).

Now, by using the proposed approach LSM based on the control point of the Wang Ball curve, we compute the approximate solution with degree 5 and degree 8 .

First, suppose that the Wang Ball curve of degree 5 is the approximate solution of 4 th order ODE with boundary conditions as follows:

$$
\begin{gathered}
y_{a}(x)=A_{a}(x)=\sum_{j=0}^{5} A_{j}^{m}(x) w_{j} \\
y_{a}(x)=A_{a}(x)=w_{0} A_{0}^{5}(x)+w_{1} A_{1}^{5}(x)+ \\
+w_{2} A_{2}^{5}(x)+w_{3} A_{3}^{5}(x)+w_{4} A_{4}^{5}(x)+w_{5} A_{5}^{5}(x) \\
y_{a}(x)=A_{a}(x)=w_{0}(1-x)^{2}+w_{1} 2 x(1-x)^{3}+ \\
+w_{2} 4 x^{2}(1-x)^{3}+w_{3} 32 x^{3}(1-x)^{2}+ \\
+w_{4} 2 x^{4}(1-x)+w_{5} x^{2}
\end{gathered}
$$

The control point's values of Wang Ball curves computed by minimizing the objective function using LSM with the given boundary conditions $y(0)=y(1)=0$, $y^{\prime}(0)=y^{\prime}(1)=0$ and as well as solving the constrained optimization problem. The values of the Wang Ball curve's control points are as follows:

$w_{0}=0, w_{1}=0, w_{2}=0.01149686, w_{3}=0.00109319$, $w_{4}=0$, and $w_{5}=0$.

Thus, we get the approximate solution of the BVP of 4th order ODE in Example 2 by the Wang Ball curve control points' base approach by replacing these control point's values back into the equation (5.6). Thus, the approximate solution of the problem in Example 2 is found through the replacement of the control point's values of the Wang Ball curve back into the equation (4.3). So, the approximate solution based on the Wang Ball curve of degree 5 for the problem in Example 2 is as follows:

$$
\begin{aligned}
y_{a}(x) & =A_{a}(x)=0.04598744 x^{2}(1-x)^{3}+ \\
& +0.03498208 x^{3}(1-x)^{2}
\end{aligned}
$$

The graph of the approximate and exact solution is presented in the following figure 6 . 


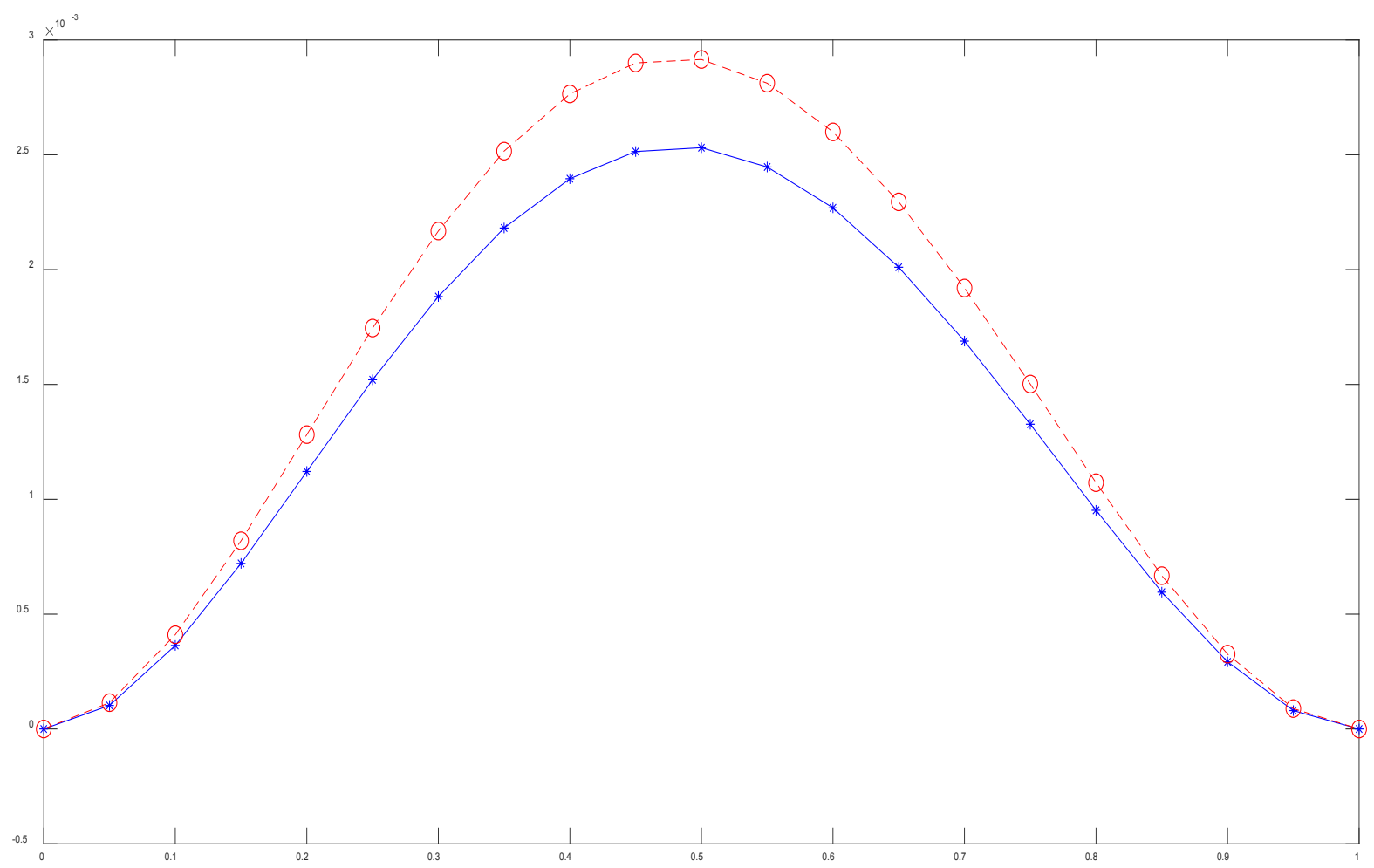

Figure 6. xact $y_{e}(x)$ (dashed with circles in red colour) and the approximate solution $y_{a}(x)$ (dashed with stars in blue colour) with Wang Ball curve's basis function of degree 5

Hence, the approximate solution is not on the desired level with degree 5. By raising the degree, we suppose that the approximate solution of the fourth order ODE is the Wang Ball curve of degree 8 as follows:

$$
\begin{gathered}
y_{a}(x)=A_{a}(x)=\sum_{j=0}^{8} A_{j}^{m}(x) w_{j} \\
y_{a}(x)=A_{a}(x)=w_{0} A_{0}^{8}(x)+w_{1} A_{1}^{8}(x)+w_{2} A_{2}^{8}(x)+ \\
+w_{3} A_{3}^{8}(x)+w_{4} A_{4}^{8}(x)+w_{5} A_{5}^{8}(x)+w_{6} A_{6}^{8}(x)+ \\
+w_{7} A_{7}^{8}(x)+w_{8} A_{8}^{8}(x) \\
y_{a}(x)=A_{a}(x)=w_{0} 2(1-x)^{2}+w_{1} 2 x(1-x)^{3}+ \\
+w_{2} 4 x^{2}(1-x)^{4}+w_{3} 8 x^{3}(1-x)^{5}+ \\
+w_{4} 16 x^{4}(1-x)^{4}+w_{5} 8 x^{5}(1-x)^{3}+ \\
+w_{6} 4 x^{4}(1-x)^{2}+w_{7} 2 x^{3}(1-x)+w_{8} x^{2}
\end{gathered}
$$

The values of control points $w_{0}, w_{1}, w_{2}, w_{3}, w_{4}, w, w_{6}, w_{7}$ and $w_{8}$ of the Wang Ball curves are found through solving the constrained optimization problem in LSM by minimizing the objective function with the boundary value conditions

$$
y(0)=y(1)=0, \quad y^{\prime}(0)=y^{\prime}(1)=0
$$

and their values are tabulated in Table 1.

Table 1. The control point's values of Wang Ball curves for Problem 2

\begin{tabular}{|c|c|}
\hline Control Points & Values \\
\hline$w_{0}$ & 0 \\
\hline$w_{1}$ & 0 \\
\hline$w_{2}$ & 0.01275270 \\
\hline$w_{3}$ & 0.01257211 \\
\hline$w_{4}$ & 0.01208323 \\
\hline$w_{5}$ & 0.01196735 \\
\hline$w_{6}$ & 0.00884018 \\
\hline$w_{7}$ & 0 \\
\hline$w_{8}$ & 0 \\
\hline
\end{tabular}

Thus, the approximate solution of the problem in Example 2 is found through the replacement of the control point's values of the Wang Ball curve back into the equation (5.8). Hence, the approximate solution found at the desired level based on the Wang Ball basis function of degree 8 for the two-point boundary value problem in Example 2 is given below:

$$
\begin{gathered}
y_{a}(x)=0.0510108 x^{2}(1-x)^{4}+ \\
+0.10057688 x^{3}(1-x)^{5}+0.19333168 x^{4}(1-x)^{4}+ \\
+0.0957388 x^{5}(1-x)^{3}+ \\
+0.03536072 x^{4}(1-x)^{2}
\end{gathered}
$$

The graphical illustration of the exact and approximate solution is shown in the following figure 7. 


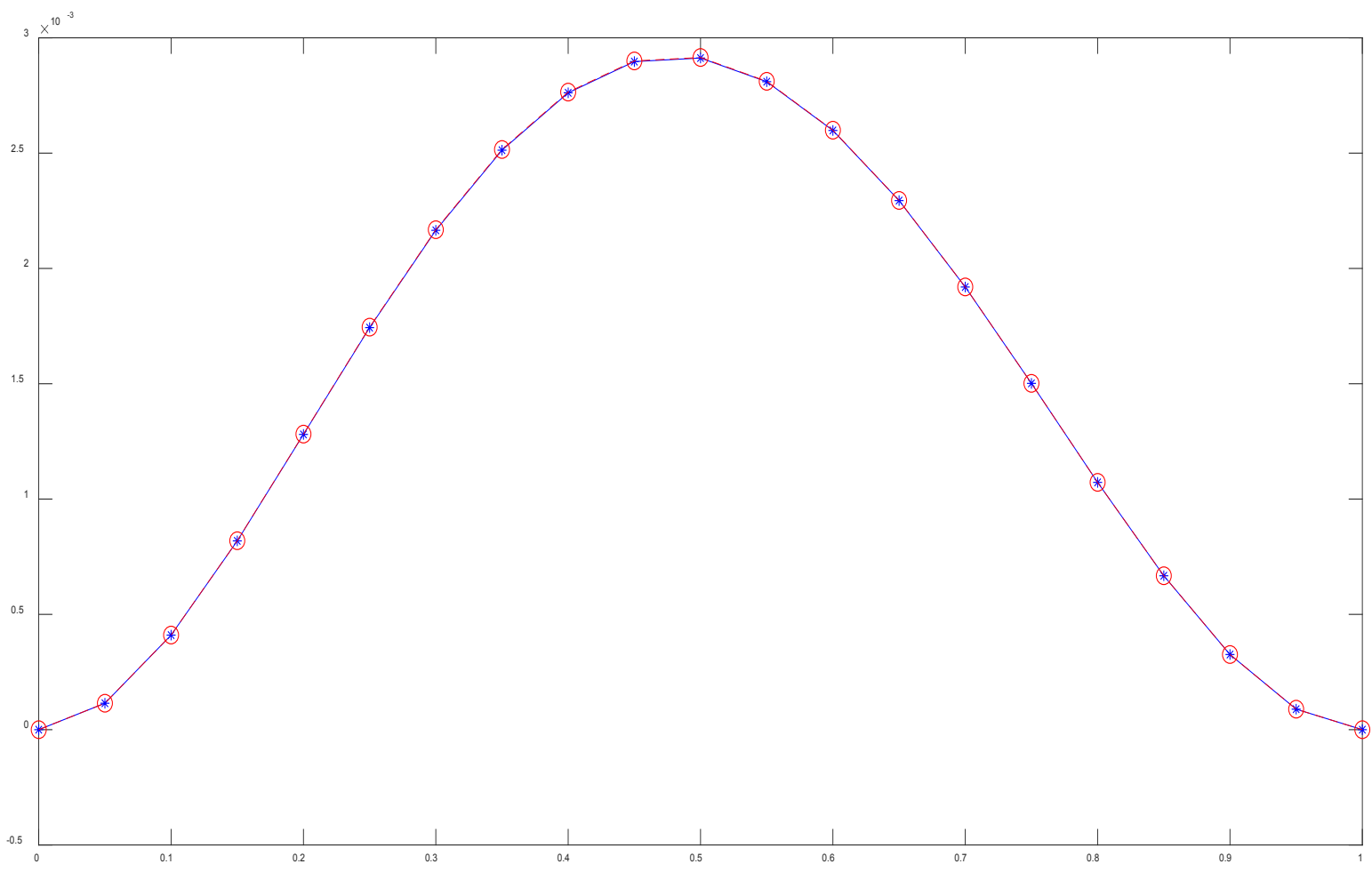

Figure 7. Exact $y_{e}(x)$ (dashed with circles in red colour) and the approximate solution $y_{a}(x)$ (dashed with stars in blue colour) with Wang Ball curve's basis function of degree 8

We solved these problems by using MATLAB based on the basis function of the Wang Ball curve. The approximate solution based on the Wang Ball basis function of degree 5 and degree 8 with the maximal error at $x=0.45$ and $x=0.40$, respectively, in the equally spaced interval $0 \leq x \leq 1$ for the problem in Example 2 is shown in Table 2 given below:

Table 2. The highest error based on the control point's values of Wang Ball curves and Bézier curve [30] for Problem 2

\begin{tabular}{|c|c|c|c|}
\hline & \multicolumn{2}{|c|}{ Errors } & Error Accuracy \\
\cline { 2 - 4 } & Highest Error & $\begin{array}{c}\text { Highest } \\
\text { Error [30] }\end{array}$ & 0.00017 \\
\hline $\begin{array}{c}\text { Basis } \\
\text { function } \\
\text { degree 5 }\end{array}$ & 0.00038 & 0.00055 & 0.0000174045 \\
\hline $\begin{array}{c}\text { Basis } \\
\text { function } \\
\text { degree 8 }\end{array}$ & 0.0000030955 & 0.0000205 & 0.005 \\
\hline
\end{tabular}

The maximal error 0.0000030955 is found between the exact and approximate solutions based on the Wang Ball basis function with degree 8 at $x=0.40 \in[0,1]$. The error accuracy is improved up to the level 0.000017404 by using LSM based on the basis function of the Wang Ball curve of degree 8 as compared to the basis function of the Bézier curve in [30].

\section{Convergence Analysis}

We conduct the convergence analysis for the proposed method based on the control points of Wang Ball curves while it's employed to the two-point boundary value problem or the higher order ODEs.

We consider the following two-point boundary value problem, which has a unique $C^{2}$ smooth solution, to analyse the convergence:

$$
\begin{gathered}
\operatorname{Lg}(x)=g^{\prime \prime} \varphi_{2}(x)+g^{\prime} \varphi_{1}(x)+g \varphi_{0}(x)=f(x), \\
0 \leq x \leq 1 \\
g(0)=a, \quad g(1)=b
\end{gathered}
$$

where $g_{j}$ and $f(x)$ are the polynomial in terms of $x$.

Based on the control points of the Wang Ball curve, we can approximate the square of $L_{2}$ norm polynomial in the interval $0 \leq x \leq 1$ through the average of the control points of Wang Ball curves.

\section{Lemma:}

If a polynomial in the form of Wang Ball curve

$$
A(x)=\sum_{j=0}^{m} w_{j, m} A_{j}^{m}(x),
$$


then:

$$
\begin{aligned}
\frac{\sum_{j=0}^{m} w_{j, m}^{2}}{m+1} & \geq \frac{\sum_{j=0}^{m+1} w_{j, m+1}^{2}}{m+2} \geq \frac{\sum_{j=0}^{m+2} w_{j, m+2}^{2}}{m+3} \\
& \geq \cdots \frac{\sum_{j=0}^{m+n} w_{j, m+n}^{2}}{m+n+1} \rightarrow \int_{0}^{1} A^{2}(x) d x, \\
n & \rightarrow+\infty
\end{aligned}
$$

where $w_{j, m+k}$ are the control points of Wang Ball curves after raising the degree to $m+\mathrm{k}$.

\section{Proof:}

By using the rule of the degree raising as

$$
w_{j, m+1}=\frac{\mathrm{j} w_{j-1, m}}{m+1}+\frac{(\mathrm{m}+1-\mathrm{j}) w_{j, m}}{m+1},
$$

hence:

$$
\begin{gathered}
\frac{1}{m+2} \sum_{j=0}^{m+1} w_{j, m+1}^{2}= \\
\frac{1}{m+2} \sum_{j=0}^{m+1}\left[\left(\frac{j}{m+1}\right)^{2} w_{j-1, m}^{2}+\left(\frac{m+1-j}{m+1}\right)^{2} w_{j, m}^{2}\right. \\
\left.+2 \frac{j(m+1-j)}{(m+1)^{2}} w_{j-1, m} w_{j, m}\right] \\
\leq \frac{1}{m+2} \sum_{j=1}^{m+1}\left(\frac{j}{m+1}\right)^{2} w_{j-1, m}^{2}+\sum_{j=0}^{m}\left(\frac{m+1-j}{m+1}\right)^{2} w_{j, m}^{2} \\
+\sum_{j=1}^{m} \frac{j(m+1-j)}{(m+1)^{2}}\left(w_{j-1, m}+w_{j, m}\right) \\
=\frac{1}{m+1} \sum_{j=0}^{m} w_{j, m}^{2}
\end{gathered}
$$

The sequence

$$
\frac{1}{m+n+1} \sum_{j=0}^{m+n} w_{j, m+n}^{2}
$$

is decreasing monotonically and it is convergent as $n \rightarrow+\infty$ because zero is its lower bound.

Now, the limit of the above sequence being equal to

$$
\int_{0}^{1} A^{2}(x) d x
$$

needs to be proven.

According to the fundamental theorem of calculus:

$$
\int_{0}^{1} A^{2}(x) d x=\lim _{n \rightarrow+\infty} \frac{1}{m+n+1} \sum_{j=0}^{m+n} A^{2}\left(\frac{j}{m+n}\right)
$$

The degree raising property of the Wang Ball curve for convergence is that there exists an arbitrary small quantity $\varepsilon_{j}, j=0,1,2,3, \ldots \ldots \ldots, m+n$ such that:

$$
\begin{gathered}
A\left(\frac{j}{m+n}\right)=w_{j, m+n}+\varepsilon_{j} \\
j=0,1,2,3, \ldots \ldots \ldots, m+n,
\end{gathered}
$$

With

$$
\lim _{n \rightarrow+\infty} \max _{j}\left|\varepsilon_{j}\right|=0
$$

Hence:

$$
\begin{aligned}
& \frac{1}{m+n+1} \sum_{j=0}^{m+n} A^{2}\left(\frac{j}{m+n}\right) \\
& =\frac{1}{m+n+1} \sum_{j=0}^{m+n}\left(w_{j, n+m}^{2}+2 w_{j, m+n} \varepsilon_{j}\right. \\
& \left.+\varepsilon_{j}^{2}\right)
\end{aligned}
$$

Let

$$
\varepsilon_{m, n}=\max _{j}\left\{\left|\varepsilon_{j}\right|\right\}_{j=0}^{m+n}
$$

Now, the last two terms of the right-hand side can be estimated as follows:

$$
\left|\frac{1}{m+n+1} \sum_{j=0}^{m+n}\left(\varepsilon_{j}^{2}\right)\right| \leq \varepsilon_{m, n}^{2}
$$

and

$$
\begin{gathered}
\left|\frac{1}{m+n+1} \sum_{j=0}^{m+n}\left(w_{j, m+n} \varepsilon_{j}\right)\right| \leq \frac{\varepsilon_{m, n}}{m+n+1} \sum_{j=0}^{m+n}\left|w_{j, m+n}\right| \\
\leq \frac{\varepsilon_{m, n}}{m+n+1} \sum_{j=0}^{m+n}\left(\frac{j}{m+n}\left|w_{j-1, m+n-1}\right|\right. \\
\left.+\frac{m+n-j}{m+n}\left|w_{j, m+n-1}\right|\right)
\end{gathered}
$$

$$
\leq \frac{\varepsilon_{m, n}}{m+n} \sum_{j=0}^{m+n-1}\left|w_{j, m+n-1}\right| \leq \cdots \leq \frac{\varepsilon_{m, n}}{m+1} \sum_{j=0}^{m}\left|w_{j, m}\right|
$$

Therefore:

$$
\begin{gathered}
\lim _{n \rightarrow+\infty} \frac{1}{m+n+1} \sum_{j=0}^{m+n}\left(2 w_{j, m+n} \varepsilon_{j}+\varepsilon_{j}^{2}\right)=0 \\
\Rightarrow \lim _{n \rightarrow+\infty} \frac{1}{m+n+1} \sum_{j=0}^{m+n} A^{2}\left(\frac{j}{m+n}\right) \\
=\lim _{n \rightarrow+\infty} \frac{1}{m+n+1} \sum_{j=0}^{m+n}\left(w_{j, n+m}^{2}\right)
\end{gathered}
$$

Hence, it is proven that the limit of the sequence is equal to

$$
\int_{0}^{1} A^{2}(x) d x
$$




\section{Theorem:}

Let $y(x)$ be the $C^{2}$ continuous solution and unique solution of two-point boundary value problem (6.1), then the approximate solution $y_{a}(x)$ be computed based on the control point of Wang Ball curves which converges to the exact solution as the degree of the approximate solution tends to infinity.

\section{Proof:}

Step 1: There exists an arbitrary small quantity $\varepsilon>0$. We obtain the polynomial $E_{S}$ with degree $S$ which satisfies the $L_{\infty}-$ norm over $0 \leq x \leq 1$, such as

$$
\left\|\frac{d^{j} E_{S}(x)}{d x^{j}}-\frac{d^{j} y(x)}{d x^{j}}\right\|_{\infty}<\frac{\varepsilon}{2}, \quad j=0,1,2
$$

by using the Weierstrass theorem [49-51] where generally, $H_{S}(x)$ does not fulfil the boundary value conditions. We can find another polynomial $G_{S}(x)=E_{S}(x)+a x+b$ with a small modification such that $G_{S}(x)$ justifies the boundary value conditions $G_{S}(0)=c$ and $G_{S}(1)=d$. Then:

$$
\left\|\frac{d^{j} G_{S}(x)}{d x^{j}}-\frac{d^{j} y(x)}{d x^{j}}\right\|_{\infty}<\varepsilon, \quad j=0,1,2
$$

Hence, the residual function can be estimated as follows:

$$
\begin{aligned}
\| L G_{S}(x) & -f(x)\left\|_{\infty}=\right\| L G_{S}(x)-y(x) \|_{\infty} \\
& \leq\left\|\varphi_{2}(x)\right\|_{\infty}\left\|G_{S}^{\prime \prime}-y^{\prime \prime}\right\|_{\infty} \\
& +\left\|\varphi_{1}(x)\right\|_{\infty}\left\|G_{S}^{\prime}-y^{\prime}\right\|_{\infty} \\
& +\left\|\varphi_{0}(x)\right\|_{\infty}\left\|G_{S}-y\right\|_{\infty} \\
& \leq\left(\left\|\varphi_{2}(x)\right\|_{\infty}+\left\|\varphi_{1}(x)\right\|_{\infty}\right. \\
& \left.+\left\|\varphi_{0}(x)\right\|_{\infty}\right) \varepsilon
\end{aligned}
$$

where $\left\|\varphi_{2}(x)\right\|_{\infty}+\left\|\varphi_{1}(x)\right\|_{\infty}+\left\|\varphi_{0}(x)\right\|_{\infty}$ is a constant.

Step 2: Now in this step, we represent the residual in the form of the Wang Ball curve as follows:

$$
R\left(G_{S}\right)=L\left(G_{S}\right)-f(x)=\sum_{j=0}^{m} w_{j, m} A_{j}^{m}(x)
$$

From the Lemma, there exists an integer $N(\geq M)$ such that if $n>N$,

$$
\begin{aligned}
& \mid \frac{1}{m+1} \sum_{j=0}^{m} w_{j, m}^{2}-\int_{0}^{1}\left(R\left(G_{S}\right)^{2} d x \mid<\varepsilon\right. \\
& \Rightarrow \frac{1}{m+1} \sum_{j=0}^{m} w_{j, m}^{2}<\varepsilon+\int_{0}^{1}\left(R\left(G_{S}\right)^{2} d x\right. \\
& \leq \varepsilon \\
&+\left[\left(\left\|\varphi_{2}(x)\right\|_{\infty}+\left\|\varphi_{1}(x)\right\|_{\infty}\right.\right. \\
&\left.\left.+\left\|\varphi_{0}(x)\right\|_{\infty}\right) \varepsilon\right]^{2}
\end{aligned}
$$

Step 3: Now, suppose that $y_{a}(x)$ is the approximate solution of (5.1) with degree $n \geq N$ which is found based on the control points of Wang Ball curves:

$$
R\left(y_{a}(x)\right)=L\left(y_{a}(x)\right)-f(x)=\sum_{j=0}^{l} c_{j, l} A_{j}^{l}(x)
$$

with degree $l \geq n \geq N$.

Further, we estimate the Sobolev norm for the difference of exact solution $y(x)$ and approximate solution $y_{a}(x)$ as follows:

$$
\begin{aligned}
\left\|y_{a}(x)-y(x)\right\|_{H^{2}[0,1]}=\int_{0}^{1} \sum_{i=0}^{2}\left|\frac{d^{i} y_{a}(x)}{d x^{i}}-\frac{d^{i} y(x)}{d x^{i}}\right| d x \\
\leq T\left(\left|y_{a}(0)-y(0)\right|+\left|y_{a}(1)-y(1)\right|\right. \\
\left.+\left\|R\left(y_{a}(x)-y(x)\right)\right\|_{2}\right) \\
=T \int_{0}^{1}\left(\sum_{j=0}^{l} w_{j, l} A_{j}^{l}(x)\right)^{2} d x \\
\leq \frac{T}{l+1} \sum_{j=0}^{l} c_{j, l}^{2}
\end{aligned}
$$

where $T$ is constant. Since the average squares of the residual of the control point of Wang Ball curves are minimized by using the control point based method, the average of the approximate solution $y_{a}(x)$ is smaller than $G_{S}(x)$.

$$
\begin{aligned}
& \text { Thus: } \\
& \qquad \begin{aligned}
\left\|y_{a}(x)-y(x)\right\|_{H^{2}[0,1]} \leq \frac{T}{l+1} \sum_{j=0}^{l} c_{j, l}^{2} \leq \frac{T}{l+1} \sum_{j=0}^{l} w_{j, l}^{2} \\
\leq \\
\leq \frac{T}{m+1} \sum_{j=0}^{m} w_{j, l}^{2} \\
\leq T\left(\varepsilon+\left(\left(\left\|\varphi_{2}(x)\right\|_{\infty}+\left\|\varphi_{1}(x)\right\|_{\infty}\right.\right.\right. \\
\left.\left.\left.+\left\|\varphi_{0}(x)\right\|_{\infty}\right) \varepsilon\right)^{2}\right)
\end{aligned}
\end{aligned}
$$

Hence, the approximate solution converges to the exact solution as the degree of the approximate solution approaches infinity.

\section{Conclusions}

The LSM is described to represent the Wang Ball basis function for the approximate solution of higher order ODEs by using the control points of Wang Ball curves. We compute the control points of Wang Ball curves through minimization of the residual function by decreasing the residual dual error. Therefore, the Wang Ball basis function presented being as the approximate solution of ODEs. We employed the Wang Ball control point base approach to approximate solution of 4 th order linear ODE with a polynomial coefficient problem. The Wang Ball basis 
function shows efficient performance in the numerical problems and a significant improvement in error accuracy than that of the Bézier curve is observed in [30]. The convergence analysis of the proposed method to the two-point boundary value problem is successfully analysed. The proposed method is Intuitive and very simple in terms of Computation, and its operation can be completed without any explicit instructions. Furthermore, as suggested in [30,47], this study recommends extending the proposed method for finding the approximate solution of other types of differential equations.

\section{REFERENCES}

[1] Nürnberger, G., \& Zeilfelder, F. (2000). Developments in bivariate spline interpolation. Journal of Computational and Applied Mathematics, 121(1-2), 125-152.

[2] Farin, G. (1997). Curves and Surfaces for Computer Aided Geometric Design-A Practical Guide (4th ed.; W. Rheinbolt, ed.). San Diego: Academic Press.

[3] Beltran, J. V., \& Monterde, J. (2004, May). Bézier solutions of the wave equation. In International Conference on Computational Science and Its Applications (pp. 631-640). Springer, Berlin, Heidelberg.

[4] Ball, A. A. (1975). CONSURF. Part two: description of the algorithms. Computer-Aided Design, 7(4), 237-242.

[5] Lai, M. J., \& Wenston, P. (2000). Bivariate spline method for numerical solution of steady state Navier-Stokes equations over polygons in stream function formulation. Numerical Methods for Partial Differential Equations: An International Journal, 16(2), 147-183.

[6] Gachpazan, M. (2011). Solving of time varying quadratic optimal control problems by using Bézier control points. Computational \& Applied Mathematics, 30(2), 367-379.

[7] Ball, A. A. (1974). CONSURF Part one: Introduction of conic lofting tile. Computer Aided Design, 6(4), 243-249.

[8] Ghomanjani, F., Farahi, M. H., \& Pariz, N. (2017). A new approach for numerical solution of a linear system with distributed delays, VOLterra delay-integro-differential equations, and nonlinear VOLterra-Fredholm integral equation by Bezier curves. Computational and Applied Mathematics, 36(3), 1349-1365.

[9] Goodman, T. N., \& Said, H. B. (1991). Shape preserving properties of the generalised Ball basis. Computer Aided Geometric Design, 8(2), 115-121.

[10] H. L. TIEN (1999) Rational Ball curves, International Journal of Mathematical Education in Science and Technology, 30:2, 243-257.

[11] Ball, A. A. (1977). CONSURF. Part 3: How the program is used. Computer-Aided Design, 9(1), 9-12.

[12] Said, H. B. (1989). A generalized Ball curve and its recursive algorithm. ACM Transactions on Graphics (TOG), 8(4), 360-371.
[13] Guojin, W. (1987). BALL CURVE OF HIGH DEGREE AND ITS GEOMETRIC PROPERTIES [J]. Applied Mathematics A Journal of Chinese Universities, 1.

[14] Hongyi, W. (2000). Unifying representation of Bézier curve and generalized Ball curves. Applied Mathematics-A Journal of Chinese Universities, 15(1), 109-121.

[15] Goodman, T. N., \& Said, H. B. (1991). Properties of generalized Ball curves and surfaces. Computer-Aided Design, 23(8), 554-560.

[16] Delgado, J., \& Pena, J. M. (2003a). A linear complexity algorithm for the Bernstein basis. International Conference on Geometric Modeling and Graphics. IEEE.

[17] Delgado, J., \& Peña, J. M. (2003b). A shape preserving representation with an evaluation algorithm of linear complexity. Computer Aided Geometric Design, 20(1), $1-10$.

[18] Aphirukmatakun, C., \& Dejdumrong, N. (2011). Multiple degree elevation and constrained multiple degree reduction for DP curves and surfaces. Computers \& Mathematics with Applications, 61(8), 2296-2299.

[19] Hu, S. M., Wang, G. Z., \& Jin, T. G. (1996). Properties of two types of generalized Ball curves. Computer-Aided Design, 28(2), 125-133.

[20] Pihien, H. N., \& Dejdumrong, N. (2000). Efficient algorithms for B ezier curves. CAGD, 17, 247-250.

[21] Jaafar, W., \& Nurhadani, W. (2018). Visualization of Curve And Surface Data Using Rational Cubic Ball Functions (Doctoral dissertation, Universiti Sains Malaysia).

[22] Aphirukmatakun, C., \& Dejdumrong, N. (2007). An approach to polynomial curve comparison in geometric object database. International Journal of Computer Science, 2(4), 240-246.

[23] Dan, Y., \& Xinmeng, C. (2007). Another type of generalized ball curves and surfaces. Acta Mathematica Scientia, 27(4), 897-907.

[24] Lambert, J. D., \& Watson, I. A. (1976). Symmetric multistip methods for periodic initial value problems. IMA Journal of Applied Mathematics, 18(2), 189-202.

[25] Hull, T. E., Enright, W. H., Fellen, B. M., \& Sedgwick, A. E. (1972). Comparing numerical methods for ordinary differential equations. SIAM Journal on Numerical Analysis, 9(4), 603-637.

[26] Sarafyan, D. (1990). New algorithms for the continuous approximate solutions of ordinary differential equations and the upgrading of the order of the processes. Computers \& Mathematics with Applications, 20(1), 77-100.

[27] Bun, R. A., \& Vasil, Y. (1992). A numerical method for solving differential equations with derivatives of any order. Computational mathematics and mathematical physics, 32(3), 317-330.

[28] Anake, T. A. (2011). Continuous implicit hybrid one-step methods for the solution of initial value problems of general second-order ordinary differential equations (Doctoral dissertation, Covenant University, Ota.).

[29] Ascher, U. (1978). Discrete Least Squares Approximations 
for Ordinary Differential Equations. SIAM J, 15(3), 478496.

[30] Zheng, J., Sederberg, W. T., \& Johnson, W. R. (2004). Least squares methods for solving differential equations using Bezier control points. Applied Numerical Mathematics, (48), 237-252.

[31] Lyche, T., \& Morken, K. (2008). Spline methods draft. Department of Informatics, Center of Mathematics for Applications, University of Oslo, Oslo.

[32] Bjorck, A. (1996). Numerical methods for least squares problems (Vol. 51). Siam.

[33] Mehrkanoon, S., Falck, T., \& Suykens, J. A. (2012). Approximate Solutions to Ordinary Differential Equations using Least Squares Support Machines. IEEE Transactions on Neural Networks and Learning Systems, 1356-1367. IEEE.

[34] Amaal A., M. (2012). Approximate Solution of Differential Equations of Fractional Orders Using Bernstein-Bézier Polynomial. Al- Mustansiriya J. Sci, 23(1), 65-74.

[35] Chistyakov, V. F., \& Chistyakova, E. V. (2013). Application of the Least Squares Method to Solving Linear Differential-Algebraic Equations. Numerical Analysis and Applications, 6(1), 77-90.

[36] Ghomanjani, F., Kamyad, A. V., \& K1lıçman, A. (2013). Bezier curves method for fourth-order integrodifferential equations. In Abstract and Applied Analysis (Vol. 2013). Hindawi.

[37] Alavizadeh, S. R., \& MAALEK, G. F. (2014). Numerical solution of higher-order linear and nonlinear ordinary differential equations with orthogonal rational legendre functions, 109-130.

[38] Yilmaz, B., \& Cetin, Y. (2017). Numerical solutions of the Fredholm integral equations of the second type. New Trends in Mathematical Sciences, 5(3), 284-292.

[39] Monterde, J. (2004). Bézier surfaces of minimal area: The Dirichlet approach. Computer Aided Geometric Design, 21(2), 117-136.

[40] Husin, S. F., Mamat, M., Asrul, M., Ibrahim, H., \& Rivaie, M. (2019). Solving Ordinary Differential Equation (ODE) Using Least Square Method: Application of Steepest
Descent Method. (5), 524-528.

[41] Fatunla, S. O. (2014). Numerical methods for initial value problems in ordinary differential equations. Academic Press.

[42] Aktas, Z., \& Stetter, H. (1977). A classification and survey of numerical methods for boundary value problems in ordinary differential equations. International Journal for Numerical Methods in Engineering, 11, 771-796.

[43] Awoyemi, D. O. (1999). A class of continuous methods for general second order initial value problems in ordinary differential equations. International Journal of Computer Mathematics, 72(1), 29-37.

[44] Sharp, P. W., \& Fine, J. M. (1992). Some Nyström pairs for the general second-order initial-value problem. Journal of computational and applied mathematics, 42(3), 279-291.

[45] Taiwo, O. A., \& Onumanyi, P. (1991). A collocation approximation of singularly perturbed second order ordinary differential equation. International journal of computer mathematics, 39(3-4), 205-211.

[46] Twizell, E. H., \& Khaliq, A. Q. M. (1984). Multiderivative methods for periodic initial value problems. SIAM journal on numerical analysis, 21(1), 111-122.

[47] [47] Evrenosoglu, M., \& Somali, S. (2008). Least squares methods for solving singularly perturbed two-point boundary value problems using Bézier control points. Applied Mathematics Letters, 21(10), 1029-1032.

[48] Husin, F. S., Mamat, M., Ibrahim, H. A. M., \& Rivaie, M. (2019). Solving Ordinary Differential Equations (ODE) Using Least Square Method: Application of Steepest Descent Method. International Journal of Recent Technology and Engineering (IJRTE), (7), 524-528.

[49] Hunter, John. "Continuous Functions." N.p., 21 July 2005. https://www.math.ucdavis.edu/ hunter/book/ch2.pdf

[50] Casselman, B. (2008). From bézier to bernstein. Feature Column from American Mathematical Society.

[51] Weierstrass, K. (1885). Über die analytische Darstellbarkeit sogenannter willkürlicher Functionen einer reellen Veränderlichen. Sitzungsberichte der Königlich Preußischen Akademie der Wissenschaften zu Berlin, 2, 633-639. 